\title{
Reliability evaluation of substation automaton system based on IEC61850
}

\author{
Li Zhiyu \\ Department of Electrical Engineering, North China Electric Power University, Baoding, China
}

Keywords: IEC6180, Substation automation system, Reliability, Evaluation

\begin{abstract}
The substation automation system based on IEC61850 standard supports free distribution of functions and its reliability is closely related to the distribution mode of its functions. The realization principles of functions' free distribution are introduced and the method of reliability evaluation of substation automation system is proposed based on IEC61850. The system's functions are deposed into the connection diagram of logical nodes based on IEC61850 which consists of logical nodes and logical connections. The fault rates of logical nodes and logical connections are calculated based on the fault rates of physical devices and physical connections, then using fault tree analysis method to calculate the availability of system's functions and using it as the reliability index of system. Finally, the interval interlock function is taken as an example to introduce the method and confirm its feasibility which provides guidance to improve the distribution mode of system's functions and enhance the reliability of system.
\end{abstract}

\section{Introduction}

To protect the security, economy, stable operation of the network, it is necessary to assess the reliability of the substation automation system. Currently, the research on the reliability analysis of substation automation system at home and abroad has focused on two aspects: First, studies on system reliability indicators; the second is based on system reliability evaluation hardware[1,2].

In this paper, the possibility of completing the intended function by the quantitative analysis system is used to assess its reliability. Based on IEC61850 standard, the substation automation system function is decomposed into logical nodes connection diagram which is consist of logical nodes and logical connections. Through the failure rate of physical devices and communication links, the failure rate of each logical nodes and logical connections can be calculated[3]. On this basis, the reliability block diagram of the logical node connection diagram according to calculate the rate of the system is available for each function, and thus assess the reliability of the system[4].

\section{Substation automation system function}

IEC61850 standard is developed by the International Electrotechnical Commission which introduces the substation automation communication networks and systems[3]. The aim of this standard is providing a way for intelligent electronic devices from different manufacturers (IED) to achieve interoperability and systems seamless integration. IEC61850 standard is totally consist of ten parts, and the Part V specifies the system functions and the communication requirements of device models. The concepts related to the system functions related are defined as follows[5,6]:

(1) Concrete function, which means the task performed by substation automation system. In general, a function consists of some logical nodes which can exchange data with each other.

(2) Physical device (PD), which is constituted by one or more processors. PD not only can receive the external resources, but also transmit data and control commands to the external device resource.

(3) Logical node (LN), which are some operations to perform a function on behalf of the physical devices. $\mathrm{LN}$ is an object defined by the data and methods, and is the smallest unit of data exchange capabilities.

(4) Distributed function, representing the function completed by two or more logical nodes located in different physical devices. 
Based on IEC61850 standard, the substation automation system function logically can be distributed on three layers: substation layer, the spacer layer and the process layer, which is including the following functions: systems support, system configuration or maintenance functions, operation or control functions, the local process automation capabilities, distributed automation support and distributed process automation functions.

\section{The assessment of system reliability}

\section{Reliability index.}

In general, the substation automation system is a repairable system, the reliability of available capacity can be described by the following characteristics:

(1) Reliability, which means normal operation probability of the equipment under the specified conditions and time, denoted by $R(t)$.

(2) Failure rate, which means the probability of the equipment occurring failure in the unit time after time, denoted by $\lambda(t)$.

(3) Mean time between failures (MTBF), which means the average working time between two failures.

(4) Repair rate, which means the probability of the equipment repaired in the unit time after time, denoted by $\mu(t)$.

(5) Mean time to repair (MTTR), which means the mean time to repair after equipment failure.

(6) Availability, which means the ability to maintain a specified function under specified conditions, denoted by $A(t)$. When $t$ is large enough, it is called a smooth availability, denoted by $A_{s}$.

When the failure rate and repair rate are constant, and the reliability satisfies the exponential distribution, ie $\lambda(t)=\lambda, \mu(t)=\mu$. Thus,

$$
\begin{aligned}
& M T B F=1 / \lambda \\
& M T T R=1 / \mu \\
& A_{s}=\frac{M T B F}{M T B F+M T T R}=\frac{\mu}{\lambda+\mu}
\end{aligned}
$$

In this paper, the reliability of the system is evaluated through calculating the smooth availability of system function.

\section{Reliability assessment.}

Physical device is consisted of one or more reliability units, and the logical node is corresponding to a certain unit of the physical device. Therefore, based on the failure rate of the physical device unit, the failure rate of its corresponding logical nodes can be calculated.

The intervals interlock function is taken as an example to describe the proposed assessment method. Based on IEC61850 standard, interval interlock function decomposition logical nodes connection diagram is shown in Fig.2, mainly including five LNs (IHMI, CSWI, CILO, XCBR, XSWI) and four LCs (XSWI-CILO, IHMI-CSWI, CSWI-XCBR, XCBR-CILO).

Assuming the failure rate of engineer workstations, interval control unit, circuit breakers, isolation switch, and physical connections are respectively $\lambda_{1}, \lambda_{2}, \lambda_{3}, \lambda_{4}, \lambda_{5}$. Thus, the failure rates of IHMI, CSWI, CILO, XCBR, XSWI are respectively $\lambda_{1}, \lambda_{2}, \lambda_{2}, \lambda_{3}, \lambda_{4}$. According to Eq.(1), the corresponding MTBF can be calculated as $1 / \lambda_{1}, 1 / \lambda_{2}, 1 / \lambda_{2}, 1 / \lambda_{3}, 1 / \lambda_{4}$. Similarly, the MTBF of XSWI-CILO, IHMI-CSWI, CSWI-XCBR, XCBR-CILO is $1 / \lambda_{5}$. The MTBF of internal physical connection of interval control unit related to CILO-CSWI is $1 / \lambda_{2}$. According to the reliability analysis method and Eq.(3), the smoothly available rate of interval interlock function is calculated as follows: 


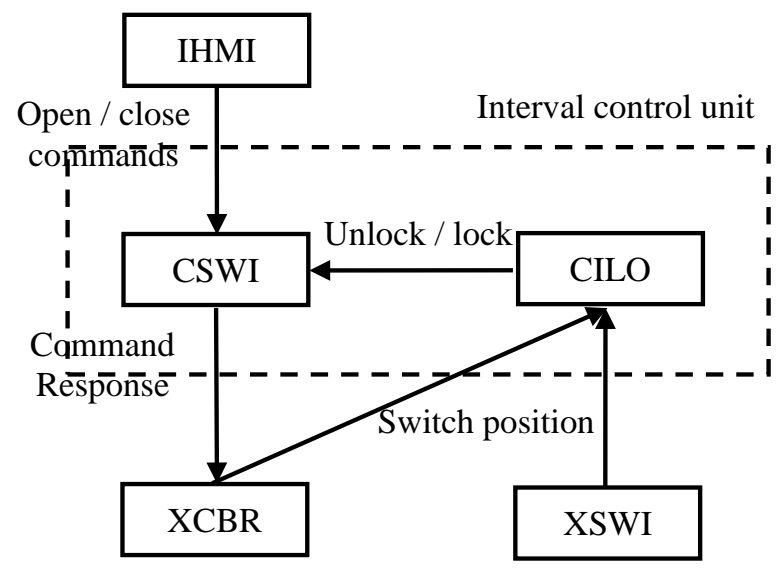

Fig.2 Logical nodes diagram of interval interlock function

$$
A_{f}=\frac{\mu_{s}^{10}}{\left(\lambda_{1}+\mu_{s}\right)\left(\lambda_{2}+\mu_{s}\right)^{3}\left(\lambda_{3}+\mu_{s}\right)\left(\lambda_{4}+\mu_{s}\right)\left(\lambda_{5}+\mu_{s}\right)^{4}}
$$

\section{Case study}

The transmission substation with T1-1 is taken as an example, including the relevant protection system operation and control functions. The basic structure of T1-1 type transmission substation is shown in Fig.3.

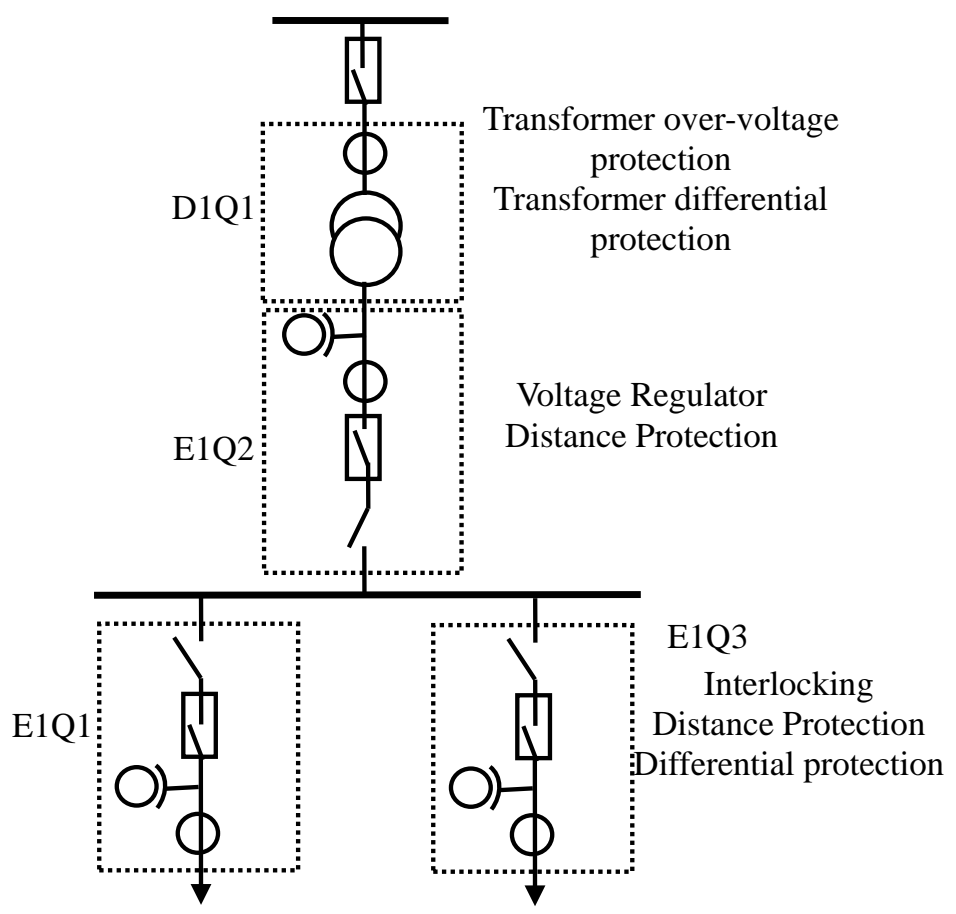

Fig.3 Structure of T1-1 substation and its protection and control functions

Based on IEC61850, the logical nodes connection diagram after decomposed from system function is shown in Fig 4.

Distribution of each logical nodes are as follows: Monitoring stations (IHMI, CALH), Remote workstation (ITCI, ITMI), Differential protection unit (PLDF), Distance protection unit (PDIS), Transformer protection and control unit (PTOV, PIOV, ATCC), Interval control unit (CILO, CSWI), Tap (YLTC), Breaker (XCBR), Disconnect switch (XSWI), Current transformer (TCTR), Voltage transformer (TVTR), Logical connection (LC).

According to the distribution of logical nodes, the use of secondary equipment manufacturers and operation and maintenance of the data to calculate the failure rate and MTBF for each logical nodes and logical connections, as shown in Table 1. 


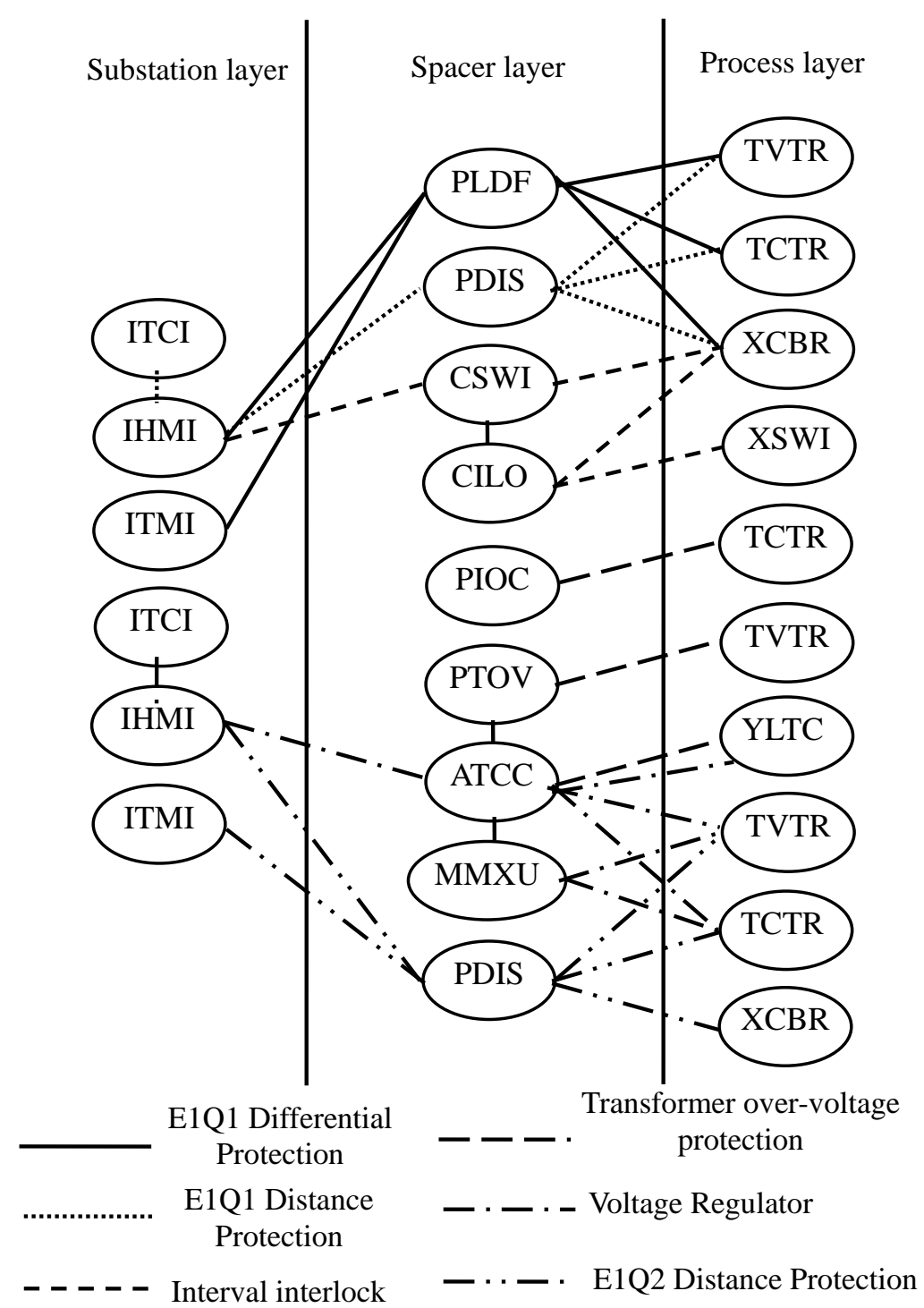

Fig.4 Logical nodes diagram of system function after being decomposed

Assuming that the MTTR of substation automation system is $4 \mathrm{~h}$. Intervals interlock function is taken as an example. According to the MTBF of LN and LC shown in Table 1 and Eq.(4), the availability rate of interval interlock function is calculated as 99.92\%. Correspondingly, the availability rate of other functions in the system and the overall system function can also be required, E1Q1 differential protection: 99.92\%, E1Q1 distance protection: 99.92\%, Transformer protection: 99.94\%, Voltage regulation: 99.90\%, E1Q2 distance protection: $99.92 \%$, The overall availability of the system functions: $99.53 \%$.

Table 1 Failure rates and MTBF of logical nodes

\begin{tabular}{ccccccc}
\hline Logical node & TVTR & TCTR & PTOV & PIOC & PLDF & PDIS \\
Failure rate & 3.62 & 3.62 & 9.08 & 9.08 & 9.08 & 9.08 \\
& 27639 & 27639 & 11015 & 11015 & 11015 & 11015 \\
MTBF/h & 7 & 7 & 7 & 7 & 7 & 7 \\
& CSWI & CILO & ATCC & YLTC & XCB & XSWI \\
Logical node & R & \\
Failure rate & 12.53 & 12.53 & 9.08 & 2.22 & 1.40 & 1.40 \\
MTBF/h & 79809 & 79809 & 11015 & 45126 & 71326 & 71326 \\
& & & 7 & 3 & 8 & 8 \\
\hline Logical node & IHMI & ITCI & ITMI & LC & & \\
Failure rate & 42.56 & 33.54 & 33.54 & 34.40 & & \\
MTBF/h & 23497 & 29816 & 29816 & 29939 & & \\
\hline
\end{tabular}


As can be seen from the system reliability evaluation process and the results, substation computer and communication links have a large influence on the availability rate of system function. Meanwhile, it is the weak link in the substation automation system. Thus, it is necessary to adopt redundant measures. A reasonable distribution way of the system functions can effectively improve the reliability of the system.

\section{Conclusion}

This paper presents a reliability assessment method for substation automation system based on IEC61850 standard, and demonstrated its feasibility by example. The proposed method evaluates the system reliability through calculating the availability rate of system function. Meanwhile, a quantitative analysis is implemented to evaluate the reasonable degree of system function distribution way, which provides guidance to improve the distribution mode of system function and enhance the reliability of system.

\section{References}

[1] Hamze Hajian-Hoseinabadi. Reliability and component importance analysis of substation automation systems [J]. International Journal of Electrical Power \& Energy Systems, 2013, 49: 455-463.

[2] Hamze Hajian-Hoseinabadi, Mohamad Esmail Hamedani Golshan, Heydar Ali Shayanfar. Composite automated distribution system reliability model considering various automated substations [J]. International Journal of Electrical Power \& Energy Systems, 2014, 54: 211-220.

[3] M. García-Gracia, S. Borroy, L. Giménez de Urtasun, M.P. Comech. Novel protection scheme based on IEC61850 [J]. Electric Power Systems Research, 2011, 81(12): 2178-2187.

[4] Otto Preiss, Alain Wegmann. Towards a composition model problem based on IEC61850 [J]. Journal of Systems and Software, 2003, 65(3): 227-236.

[5] Jiping Lu, Wenyuan Li, Wei Yan. State enumeration technique combined with a labeling bus set approach for reliability evaluation of substation configuration in power systems[J]. Electric Power Systems Research, 2007, 77(5): 401-406.

[6] B. Retterath, A.A. Chowdhury, S.S. Venkata. Decoupled substation reliability assessment[J]. International Journal of Electrical Power \& Energy Systems,2005, 27(9): 662-668. 